\title{
Decades Changes in Prevalence of Disability and Comorbidities'Impact on Elderly with Diabetic in China: Multivariate Decomposition Analysis of Chinese Longitudinal Healthy Longevity Survey Data
}

Shaohong Liao

Shandong Technology and Business University https://orcid.org/0000-0002-3181-9161

Chunling Song ( $\nabla$ cl-song@sohu.com )

Shandong Technology and Business University https://orcid.org/0000-0001-6171-0848

\section{Research}

Keywords: Comorbidities in diabetic, Disability, Multivariate decomposition method, Dynamic equilibrium

Posted Date: March 11th, 2021

DOl: https://doi.org/10.21203/rs.3.rs-282685/v1

License: (c) (1) This work is licensed under a Creative Commons Attribution 4.0 International License.

Read Full License 


\title{
Decades Changes in Prevalence of Disability and Comorbidities' Impact on Elderly with Diabetic in China: Multivariate Decomposition Analysis of Chinese Longitudinal Healthy Longevity Survey Data
}

Shaohong Liao ${ }^{1}$ and Chunling Song ${ }^{2 *}$

\begin{abstract}
Background: In China, the prevalence of diabetes is increasing and the prevalence of disability in the elderly population is decreasing. However, few studies have analyzed the change of ADL or IADL disability and the influence of comorbidity in elderly patients with diabetes in China using national data.
\end{abstract}

Methods: Based on data from Chinese Longitudinal Healthy Longevity Survey (CLHLS) from 2008 to 2018, this study not only explored the trend of Chinese elderly prevalence of diabetes and disability but using multivariate decomposition method analyzed the influence of comorbidity in patient's disability with diabetes between 2008 and 2018.

Results: The prevalence of diabetes among the elderly in China increased from 4.2 percent in 2008 to 13.5 percent in 2018, with a larger increase among women, and the prevalence of diabetes in the elderly was lower than that in the younger age group. For older adults with diabetes, the estimated likelihood of ADL having at least one difficulty decreased from 8.3 percent in 2008 to 6.3 percent in 2018. The proportion of women with diabetes with at least one difficulty in IADL increased and decreased in men. The decrease of underweight contributed the most to the decrease of ADL and IADL in men, accounting for $52.8 \%$ and $61.4 \%$, respectively. The decrease in the proportion of somatic comorbidities, underweight, and negative emotions was associated with a decrease in the likelihood of having at least one difficulty in ADL in women with diabetes $(14.8 \%, 23.8 \%$, and $30.3 \%$, respectively). Comorbidities had no significant effect on the increased probability of women having at least one difficulty with IADL.

Conclusions: Although the prevalence of diabetes in older Chinese adults increased over time, the proportion of male and female adults with at least one difficulty in IADL decreased over time, except for a slight increase in the proportion of female adults in IADL. The proportion of underweight and negative emotion in diabetic patients showed a decreasing trend, which to a certain extent inhibited the negative impact of the increase in the prevalence of somatic comorbidities and elderly disability. The results of this study partially support the "dynamic equilibrium" hypothesis of diabetes incidence.

Keywords: Comorbidities in diabetic, Disability, Multivariate decomposition method, Dynamic equilibrium

\footnotetext{
1 East Asian Institute for Social Development, Shandong Technology and Business University, Yantai, China, No.191 Binhai Middle Road, Yantai City 264005, China

2 Public School, Shandong Technology and Business University, Yantai, China, No.191 Binhai Middle Road, Yantai City 264005, China

*Correspondence: cl-song@sohu.com
} 


\section{Introduction}

The global spread of COVID-19 in 2020 presents a comprehensive challenge to public health prevention. The 2019 global burden of disease showed that disability has become an increasingly large share of the global disease burden, rising from $21 \%$ of the total burden in 1990 to $34 \%$ in 2019 [1. . Age-related syndromities (e.g., falls, frailty, cognitive impairment) and chronic diseases (e.g., high blood pressure, myocardial infarction, arthritis, diabetes, stroke) play an important role in disability [2. ]. This makes the elderly the most vulnerable group to health risks such as those associated with the Covid-19 pandemic.

In recent years, many studies showed a worldwide trend of declining trend of disability and increasing life expectancy in old age [3. -5. ]. A study showed that prevalence of ADL with at least one difficulty among the elderly with diabetes in Germany increased significantly from $11.3 \%$ in 2004 to $19.1 \%$ in 2015. The incidence of multimorbidity diseases promoted the incidence of disability [6. . Many studies on China have shown that the changes of diseases among the elderly in China conform to the hypothesis of "disease expansion" [7., which means that while the life expectancy of the elderly in China increased, the duration time of suffering from diseases and prevalence of disability increased too [8. ]. According to WHO criteria, the weighted prevalence of adult diabetes diagnosed in China increased from $9.7 \%$ in 2007 and 2010 in $10.4 \%$ to $11.6 \%$ in 2013 [9. 11. ], and further reached $11.2 \%$ in 2017, using 2018 diagnostic criteria from the American Diabetes Association [12. ]. With the increasing prevalence of diabetes, the Chinese government was aware that diabetes has become a very important public health problem. In 2019, the State Council of the People's Republic of China issued the Healthy China initiative to implement the diabetes prevention and treatment action, prompting residents to pay attention to blood sugar levels, guiding people with pre-diabetes to scientifically reduce the risk of the disease, guiding diabetics to strengthen health management, delay or prevent the occurrence and development of diabetes, and improving the diagnosed rate of diabetes [13. ]. From prevention perspective, the Chinese government tried its best to reduce the public health costs associated with the rising incidence of diabetes.

In China, the rise in diabetes prevalence coincides with a decline in the prevalence of disability in the elderly population [14. 15. ]. Some studies have shown that the ADL disability rate of the elderly in China decreased from $11.08 \%$ in 2011 to $10.26 \%$ in 2015 [16. ]. Other studies have shown that the incidence of ADL in the elderly aged 65 and above in China decreased from $6.42 \%$ in 2002 to $4.66 \%$ in 2014 [17. ]. Although the overall disability rate is decreasing, few studies have analyzed the change of ADL or IADL disability rate in elderly diabetic patients in China. Therefore, we need to further explore the changing trend of diabetes prevalence from 2008 to 2018 and analyze the changing characteristics and influencing factors of prevalence of ADL and IADL disability in diabetic patients. If the increase in the prevalence of diabetes among the elderly can be offset by the decrease in disability, this would be consistent with the hypothesis of "dynamic equilibrium" [18. ] and would have more positive implications for the chronic disease management policies currently being adopted in China.

\section{Data and Methods}

\section{Data source}

The data used in this study were from the Chinese Longitudinal Healthy Longevity Survey (CLHLS) managed by the Research Center for Health and Aging Development at Peking University. It is a 
senior citizen tracking survey organized by the Research Center on Healthy Aging and Development of Peking University/National Development Institute. The survey covers 23 provinces, municipalities and autonomous regions in China. The project was followed up in 2000, 2002, 2005, 2008-2009, 2011-2012, 2014 and 2017-2018 after conducting a baseline survey in 1998. In the follow-up survey in 2008-2009, 9,088 samples were added, and in the follow-up survey in 2017$2018,12,176$ respondents were added. In order to eliminate the possible influence of individual repetition on the results in the two surveys, the individual data tracked in the 2017-2018 survey were deleted from the eighth survey, and the final respondents included 16,563 and 13,363 elderly people aged $65+$.For more details on CLHLS study design, sampling, measurement, and data quality see Zeng et al. [19. ].

\section{Measurement}

The measurement of disability. The self-care ability scale developed by Katz [20. ] is generally used internationally to measure the six ADLs and IADL index [21. ] to measure the instrumental daily activities. CHLHS provides six ADLs (including dressing, bathing, eating, fluctuation bed, toilet and control urine) and eight IADLs (able to go outside to visit neighbors, go shopping by yourself, make food by yourself, wash clothes, walk one kilometer, carry $5 \mathrm{~kg}$ weight, crouch and stand three times, Take public transportation). They are divided into " Yes, very limited"(1 point); "Yes, to some extent limited"( 2 points); "Yes, I have difficulty and need help" ( 3 points), or "I cannot do it" (4 points). Having difficulty and need help or being unable to do at least one of the six ADLs or eight IADLs was defined as a disability. The dummy variables of "ADL/IADL" were encoded as 1 , and 0 otherwise.

Measurement of diabetes mellitus and comorbidities. Chronic disease prevalence in older adults was investigated in CLHLS, and diabetes was primarily self-reported rather than medically diagnosed. Respondents were asked whether they had diabetes, and "yes" was to confirm that they had diabetes, while "no" was to confirm that they did not have diabetes. The samples of individuals who answered "don't know" or didn't answer were removed. The presence of somatic comorbidities of diabetes was measured by the number of self-reported cases of 14 other chronic diseases. If the number of other chronic diseases is not less than 2 , it is considered to have somatic comorbidities; if the total number is less than 2, it is 0 ; otherwise, it is 1 . The 14 chronic diseases include high blood pressure, heart disease, stroke and cerebrovascular disease, bronchitis, emphysema, asthma or pneumonia, tuberculosis, cataracts, glaucoma, cancer, prostate disease, gastrointestinal ulcers, Parkinson's disease, bedsores, arthritis and dementia.

Negative emotions: although there was a depression and anxiety scale in the CLHLS 2018 survey, there was no complete depression scale in the 2008 survey. Therefore, in order to ensure the consistency of the data, positive emotions were assessed by three questions [22. ]: "Do you usually feel nervous or fearful?", "Do you often feel lonely?", and "Do you feel increasingly useless in general?" The answer to each question is somewhere between "never" (coded as 5) and "always" (coded as 1). We aggregate scores from three questions (between 3 and 15) to form an overall score, with high scores indicating good emotional state. A total score of less than 9 is considered to have certain negative emotions, set as 1 and 0 for others.

BMI. BMI is calculated by dividing weight $(\mathrm{kg})$ by height $(\mathrm{M})$ squared. Studies conducted by some scholars on China have shown that when BMI is classified according to Chinese guidelines, the risk of ADL difficulty is significantly reduced in the elderly population over 80 years old with a higher BMI (BMI>24), but the risk of ADL difficulty is significantly increased in the low BMI (BMI 
$\leqslant 18.5$ )[23. ].Based on this conclusion, in order to ensure the suitability of the analysis results, BMI was recoded to generate two new variables. When BMI is no higher than 18.5, it means Underweight and is represented by 1 , while the others are 0. Overweight is expressed by 1 if BMI is above 24, while the others are 0 .

Covariates. The analysis included two groups of covariates related to functional ability. The first group of covariates included indicators of demographic characteristics, such as age, sex (male code 1), marital status (spouse or cohabitant code 1) and rural/urban residence (urban code 1). There are two categories of age: 65 to 79 and over 80.A second set of covariates, including economic status through education and self-reported, can be used to measure an individual's socioeconomic status. Given the relatively low level of education of the elderly in China, education can be divided into two categories: illiterate (code 0 ) and literate (code 1). Individual economic status was assessed by "How do you rate your own economic status (compared to other local people)", with variables in three categories: poor, average and rich.

\section{Statistical analysis method}

Estimation of disability probability. We estimated the probability of diabetes in both men and women by using logistic regression for age, education, marital status, residence, and economic status. Stratified by gender and year of investigation, similar models were used to estimate the disability probabilities of patients with diabetes and those without diabetes. When predicting the probability, the mean value of each impact factor is assigned as the variable, and the mean value remains unchanged in the two tracking surveys. We also reported changes in the proportion of ADL and IADL in the "young and elderly population (aged 65-79 years)" and the "elderly population (aged 80 years and above)".

Oaxaca-Binder decomposition technique. We used the Oaxaca-Binder decomposition technique to explain the effects of somatic comorbidities, weight loss, overweight, and negative emotions on ADL and IADL changes in Chinese elderly diabetic patients from 2008 to 2018. The Oaxaca-Blinder decomposition method decomposes the changes (differences) in ADL or IADL of diabetic patients in 2018 and 2008 into "E" and "C", where "E" represents the changes caused by differences in individual endowment or characteristics between the 2008 survey and the 2018 survey, which can be explained by observed covariates. " $\mathrm{C}$ " refers to the part of the change (difference) due to the difference in the coefficient or effect, usually called the unexplained part or the coefficient effect. In this paper, it refers to the changes in the degree to which variables such as somatic comorbidities, weight loss, overweight and negative emotions influence ADL or IADL difficulty.

The multivariate decomposition for nonlinear response models (MDNRM) developed by Powers et al. [24. ] is adopted for decomposition. In 2008-2018, the nonlinear logit Oaxaca-Blinder decomposition of ADL and IADL differences in elderly diabetic patients can be written as follows:

$$
\bar{y}^{A}-\bar{y}^{B}=\left[F\left(X^{\bar{A}} \hat{\beta}^{A}\right)-F\left(X^{\bar{B}} \hat{\beta}^{A}\right)\right]+\left[F\left(X^{\bar{B}} \hat{\beta}^{A}\right)-F\left(X^{\bar{B}} \hat{\beta}^{B}\right)\right]
$$

Subscripts $A$ and $B$ represent elderly diabetic patients investigated by Wave2018 and Wave2008. $\bar{y}$ denotes the average of the results, $\hat{\beta}$ denotes the coefficient estimated with Logit model, and $F(\cdot)$ denotes the cumulative distribution function of logistic distribution. The first square bracket measures the change due to characteristic differences (" characteristic effects "). If the " characteristic effect" coefficient is positive, this means that the expected ADL or IADL difference will be reduced if the covariables of Wave 2018 follows the same distribution as the covariables of Wave 2008.The second square bracket measures the difference due to the different effects of observed features (" coefficient effects ").A negative coefficient on the "coefficient effect" 
means that the expected difference in ADL or IADL of Wave 2008 would increase if the individual ADL or IADL of Wave 2018 responded to the covariables to the same degree as the individual Wave 2018.

\section{Results}

Changes in Predicted Probabilities of Diabetes between Waves 2008 and 2018

Compared the changes of the analysis of features of diabetic individuals with diabetes, can be found that in the diabetic population in the proportion of women than men, with an average age of lower literacy, urban living, have a spouse, the higher percentage of self-reported economy is rich in (table 1). The estimated prevalence of diabetes increased from 4.2 percent in 2008 to 13.5 percent in 2018. By sex, the estimated risk of diabetes increased from $3.2 \%$ to $11.1 \%$ for men and from $4.8 \%$ to $15.5 \%$ for women. In terms of age groups, the probability of diabetes in the elderly was lower than that in the younger elderly, but it increased significantly in 2018 compared with 2008, and the increase rate in women was greater than that in men (Table 2).

Table 1 Weighted Sample Characteristics in \%

\begin{tabular}{lcccc}
\hline & \multicolumn{2}{c}{ Without Diabetes } & \multicolumn{2}{c}{ With Diabetes } \\
& $2008(\mathrm{n}=15807)$ & $2018(\mathrm{n}=10692)$ & $2008(\mathrm{n}=422)$ & $2018(\mathrm{n}=1218)$ \\
\hline $\begin{array}{l}\text { Gender } \\
\text { Male }\end{array}$ & 48.26 & & & \\
$\quad$ Female & 51.74 & 59.53 & 40.93 & 39.99 \\
Averaged age & & 50.47 & 59.07 & 60.01 \\
$\quad$ Male & 72.70 & 71.98 & 71.68 & 71.67 \\
$\quad$ Female & 73.64 & 72.66 & 72.85 & 71.93 \\
Age & & & & \\
65-79 & 83.57 & 84.62 & 88.54 & 86.89 \\
$\quad$ 80+ & 16.43 & 15.38 & 11.46 & 13.11 \\
Education & & & & \\
$\quad$ Illiteracy & 43.09 & 26.09 & 32.11 & 23.08 \\
Literacy & 56.91 & 73.91 & 67.89 & 76.92 \\
Residence & & & & \\
$\quad$ Urban & 41.84 & 47.76 & 69.64 & 66.46 \\
$\quad$ Rural & 58.16 & 52.24 & 30.36 & 33.54 \\
Spouse & & & \\
$\quad$ With Spous & 62.63 & 72.29 & 66.15 & 74.97 \\
$\quad$ Without spouse & 37.37 & 27.71 & 33.85 & 25.03 \\
Self-reported economic status & & & & \\
$\quad$ Rich & 12.83 & 18.27 & 14.78 & 19.63 \\
Average & 71.50 & 71.29 & 71.18 & 68.88 \\
$\quad$ Poor & 15.66 & 10.43 & 14.04 & 11.49 \\
\hline
\end{tabular}


Table 2 Change in Diabetes Prevalence between Wave 2018 and Wave 2008 Stratified by Gender

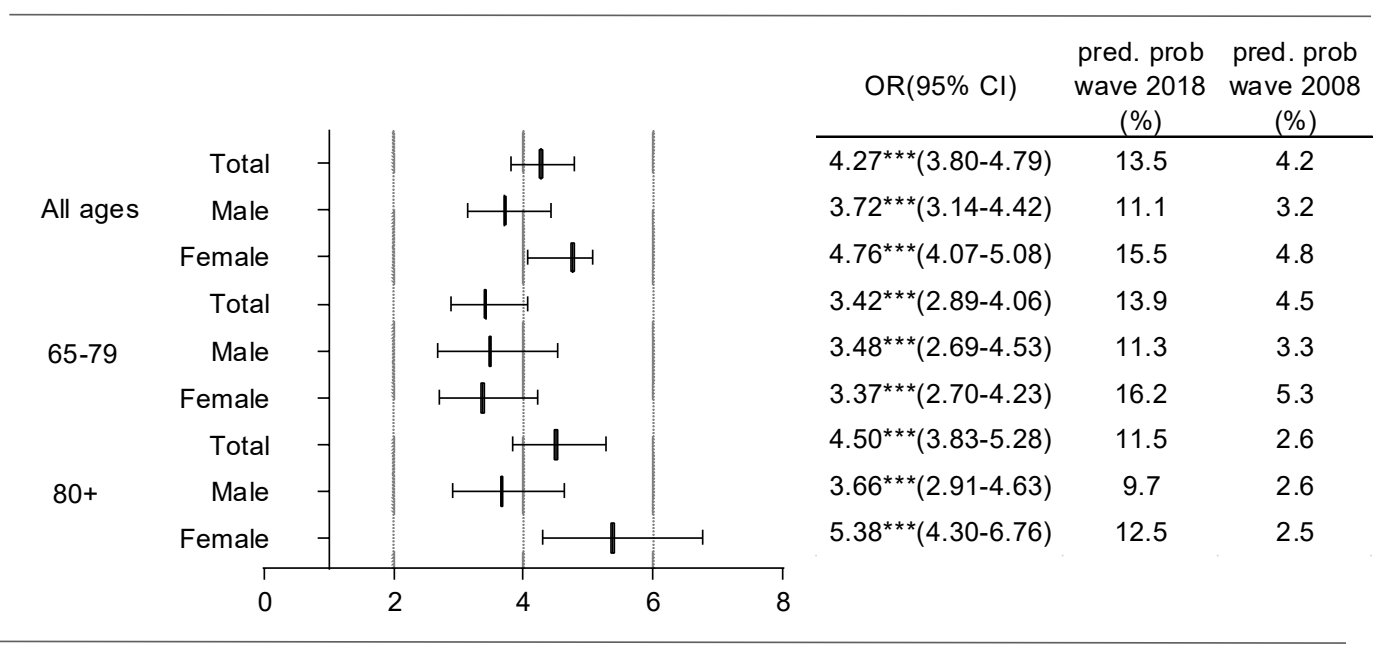

Note: Adjusted for age, education, married status and residence; $\mathrm{OR}=$ odds ratio, reference wave 2008; \% = predicted probabilities; ***p <.001; CLHLS 2008 and 2018.

Whether individuals with diabetes have at least one difficulty ratio of ADL to IADL. For individuals with diabetes, it is estimated that the probability of having at least one difficulty in ADL decreased from $8.3 \%$ in 2008 to $6.3 \%$ in 2018 , and both decreased (Table 3). The IADL difficulty of diabetic patients generally increased slightly, but decreased in male diabetic patients, which was statistically significant (Table 4). According to age, Except for male diabetic patients aged 80 and above, the estimated probability of ADL with at least one difficulty decreased. For individuals without diabetes, it is estimated that the probability of at least one ADL and IADL difficulty had increased, but it was still far lower than that of diabetic patients (Figure 1).

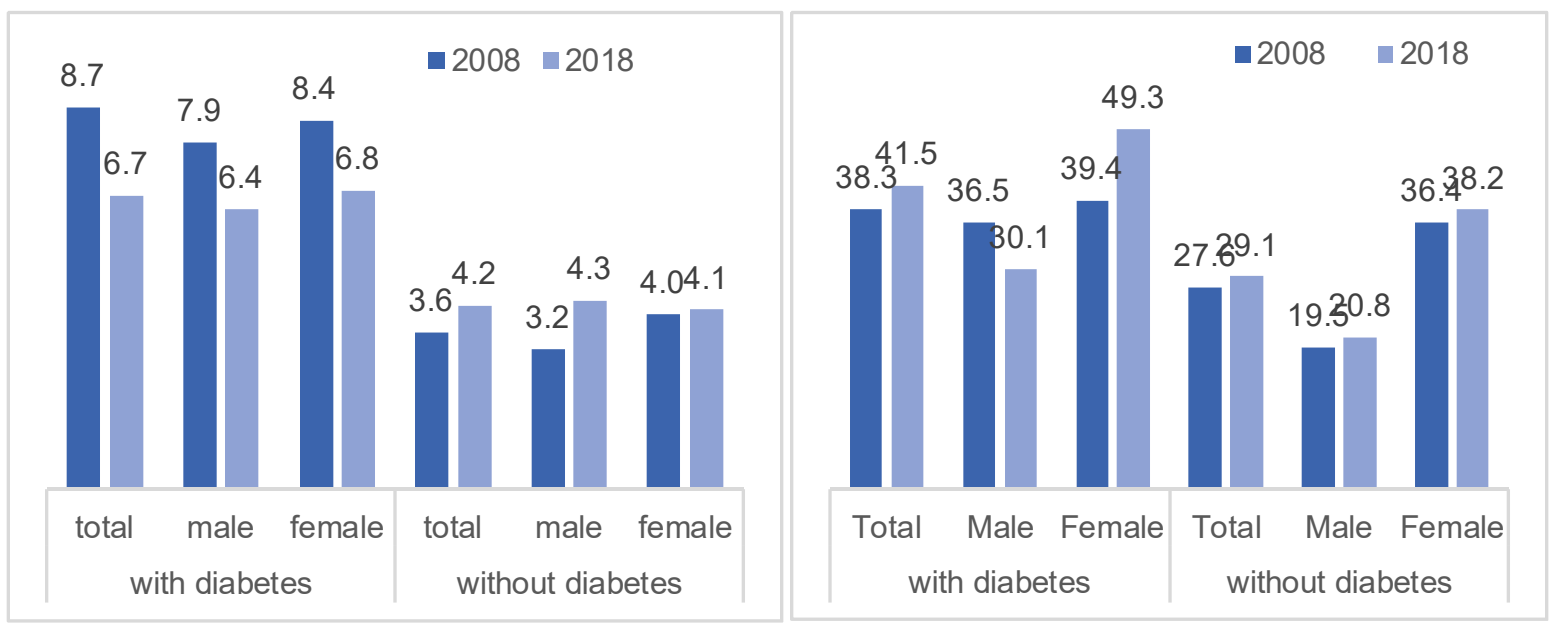

Figure 1 Change in ADL and IADL between 2008 and 2018 among people with and without diabetes, predicted probabilities in \%. Adjusted for age, education, married status and residence, for the bars "total" also for gender, CLHLS, 2008 and 2018. ADL = activities of daily living. IADL = instrumental activities of daily living 
Table 3 Change in ADL and IADL with Diabetes between two Waves Stratified by Gender and Age

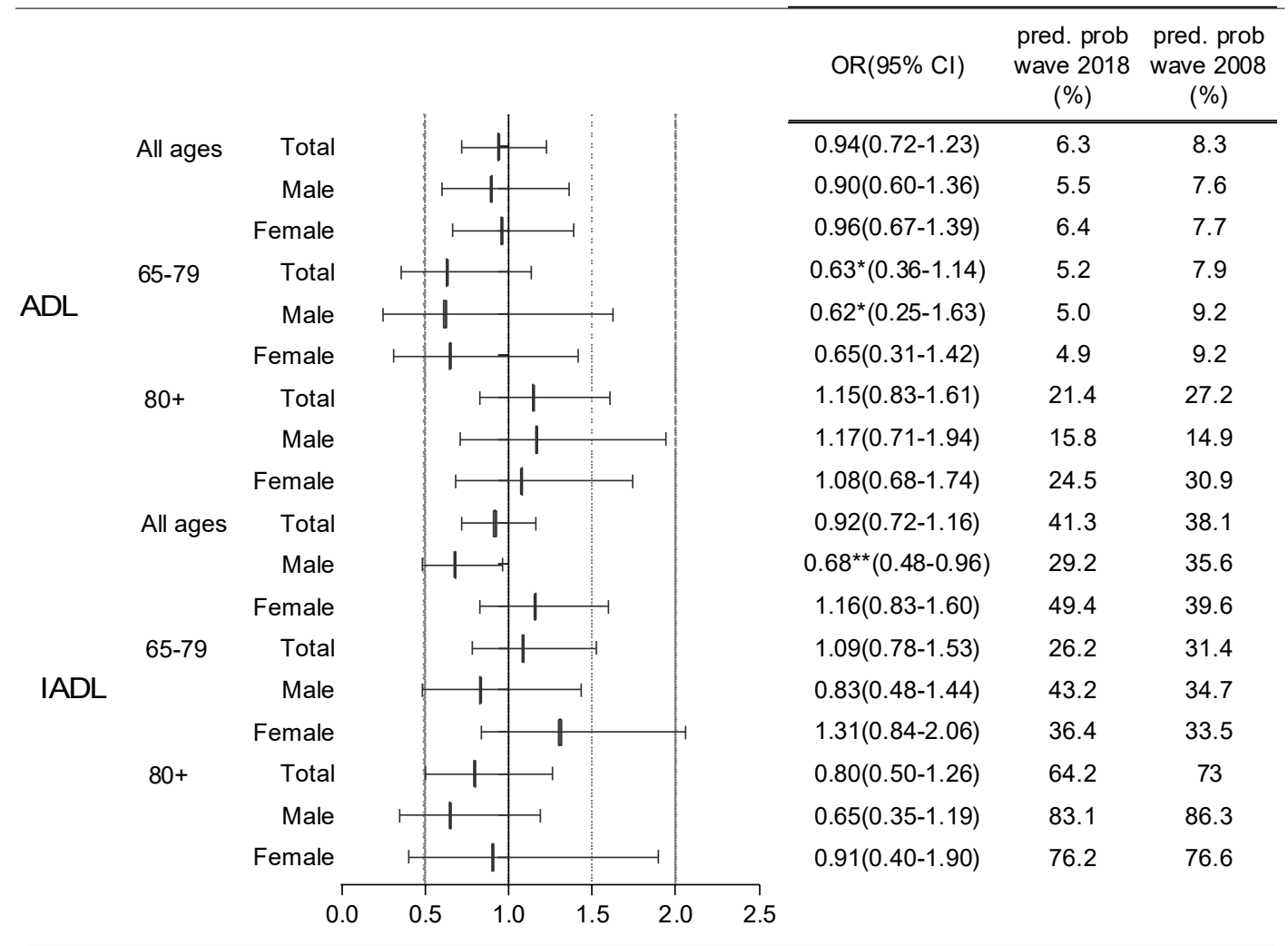

Note. Adjusted for age, education, married status and residence; $\mathrm{OR}=$ odds ratio, reference wave 2008; \% = predicted probabilities; ${ }^{* * p}<.05, * \mathrm{p}<.1 ;$ CLHLS 2008 and 2018.

\section{Changes in the characteristics and coefficients of ADL and IADL}

Compared with the characteristics of male diabetes patients in 2008 and 2018, this study found that the level of somatic comorbidities of patients in 2018 was significantly higher, the level of underweight decreased, the probability of overweight increased, and the probability of negative mood decreased slightly. Except for somatic comorbidities, all the others reached statistical significance. Changes in the predictive probability of somatic comorbidities, underweight and negative emotions in female diabetic patients showed similar characteristics to those in male patients (Table 4).

From 2008 to 2018, ADL difficulty in women with diabetes decreased by 2.73 percent. Different from male patients with diabetes, the main reason was the effect of characteristic changes, accounting for $71.4 \%$ of the overall decline, which it was statistically significant. If the levels of somatic comorbidities, underweight and negative emotions in 2018 were the same as those in 2008, the probability of ADL difficulty would be significantly reduced by $0.40 \%, 0.65 \%$ and $0.83 \%$.In terms of IADL, the probability of difficulty in IADL in female diabetic patients increased by $7.90 \%$, and the difference caused by the change of coefficient significantly increased by $9.24 \%$, and the difference caused by the change of characteristics decreased by $1.34 \%$. The change of characteristics was positively affected by the decrease of "underweight" and negatively affected by the increase of the proportion of oldest-old age, respectively, and the influence of the two variables was statistically significant. 
Table 4 Change in Characteristics of Somatic Comorbidities, Underweight, Overweight, Depression between Wave 2008 and Wave 2018 among Male and Female with Diabetes

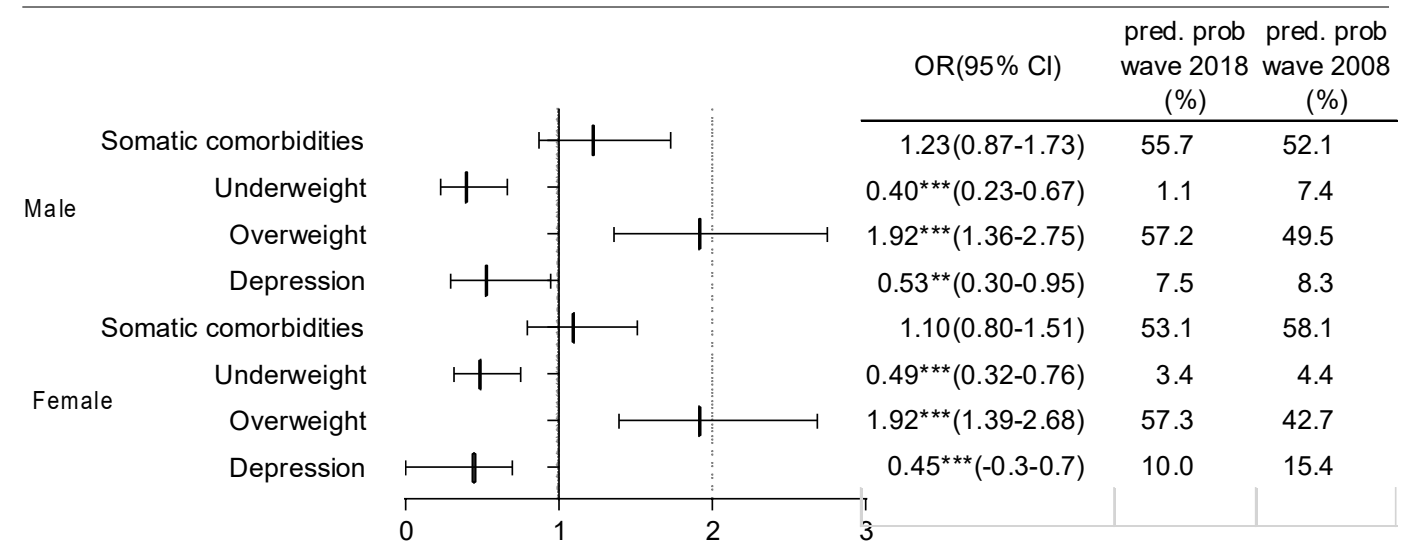

Note. Adjusted for age, education, married status and residence; OR = odds ratio, reference wave 2008; \% $=$ predicted probabilities; $* * * p<.01, * * p<.05, * p<.1 ;$ CLHLS 2008 and 2018.

As showed in Table 5, the influence of somatic comorbidities on diabetic patients with disability of ADL and IADL decreased from 2008 to 2018. However, the higher rate of somatic comorbidities, the higher prevalence of IADL and IADL with at least one difficulty in both male and female with diabetics. The effect of underweight increased and was statistically significant for Wave 2018. Overweight had no significant effect on ADL and IADL in diabetic patients. From 2008 to 2018, the effect of negative emotions on ADL decreased in men, but increased in men's IADL, decreased in women's ADL and IADL, and the effect on ADL disability was statistically significant. Excepting male ADL disability in Wave 2008, oldest-old age had a significant positive effect on ADL and IADL disability. However, from 2008 to 2018, the effect of oldest-old age on ADL and IADL disability decreased in females and increased in males.

Through MDNRM analysis, it can be found that the proportion of ADL difficulty in male diabetic patients decreased by $3.03 \%$ from 2008 to 2018 , and the most important factor was the change of coefficient, which accounted for $54.4 \%$ of the overall decrease. However, no statistically significant difference was found in the change of feature (E) and change of coefficient (C). From 2008 to 2018, the decrease of male IADL reached $5.50 \%$, of which, the change of characteristics accounted for $44.4 \%$, and the change of coefficient accounted for $55.6 \%$, and the influence of the change of characteristics was statistically significant. According to the analysis of the influence of characteristic changes, the most important influence was underweight, which accounted for $52.8 \%$ of ADL and $61.4 \%$ of IADL decrease, respectively. Thus, if the level of underweight in older men with diabetes in 2018 was the same as in 2008, the difference in the expected probability of ADL and IADL disability would widen. This suggests that a decrease in underweight in older men with diabetes in 2018 could contribute to a decrease in the probability of ADL and IADL disability. Secondly, the increase of the proportion of the elderly will promote the increase of the probability of ADL and IADL disability. Thirdly, the increase of somatic comorbidity in elderly male diabetes patients will lead to a small increase in the probability of IADL disability, and the decrease of negative emotion level will lead to a small decrease in the probability of IADL disability. 
Table 5 Effects of Somatic Comorbidities, Underweight, Overweight, Depression and Age on ADL and IADL in Wave 2008 and Wave 2018 among Male and Female with Diabetes

\begin{tabular}{cccccc}
\hline \multirow{2}{*}{ Variables } & \multirow{2}{*}{ Wave } & \multicolumn{2}{c}{ ADL } & \multicolumn{2}{c}{ IADL } \\
\cline { 3 - 6 } Somatic & Wave 2008 & 0.929 & $1.964^{* * *}$ & $1.627^{* * *}$ & $0.812^{* *}$ \\
Comorbidities & & $(0.721)$ & $(0.457)$ & $(0.489)$ & $(0.394)$ \\
& Wave 2018 & -0.0756 & $0.764^{* *}$ & $0.498^{*}$ & $0.383^{*}$ \\
& & $(0.454)$ & $(0.357)$ & $(0.270)$ & $(0.219)$ \\
\hline \multirow{3}{*}{ Underweight } & Wave 2008 & -0.287 & 0.776 & 0.980 & 1.099 \\
& & $(0.839)$ & $(0.847)$ & $(0.750)$ & $(0.839)$ \\
& Wave 2018 & $1.747^{* * *}$ & $1.783^{* * *}$ & $2.793^{* * *}$ & $1.449^{* *}$ \\
& & $(0.667)$ & $(0.537)$ & $(0.918)$ & $(0.662)$ \\
\hline \multirow{3}{*}{ Overweight } & Wave 2008 & -0.882 & 0.472 & -0.300 & -0.00632 \\
& & $(0.824)$ & $(0.564)$ & $(0.514)$ & $(0.416)$ \\
& Wave 2018 & 0.0258 & -0.256 & 0.250 & -0.00758 \\
& & $(0.455)$ & $(0.375)$ & $(0.280)$ & $(0.226)$ \\
\hline \multirow{3}{*}{ Depression } & Wave 2008 & 1.489 & $1.383^{* *}$ & 0.373 & $1.063^{* *}$ \\
& & $(0.937)$ & $(0.563)$ & $(0.978)$ & $(0.522)$ \\
& Wave 2018 & 0.127 & $1.153^{* *}$ & $1.568^{* * *}$ & 0.338 \\
& & $(0.613)$ & $(0.449)$ & $(0.472)$ & $(0.349)$ \\
\hline \multirow{3}{*}{ Oldest-old } & Wave 2008 & 0.705 & $1.742^{* * *}$ & $1.434^{* * *}$ & $1.882^{* * *}$ \\
& & $(0.583)$ & $(0.499)$ & $(0.506)$ & $(0.449)$ \\
& Wave 2018 & $1.082^{* * *}$ & $1.449 * * *$ & $1.477^{* * *}$ & $1.791^{* * *}$ \\
& & $(0.356)$ & $(0.297)$ & $(0.244)$ & $(0.227)$ \\
\hline
\end{tabular}

se in parentheses, $* * * \mathrm{p}<0.01, * * \mathrm{p}<0.05, * \mathrm{p}<0.1$

Note. somatic comorbidities $=$ at least two additional somatic comorbidities from a list of 14 chronic somatic diseases; $A D L=$ activities of daily living; $I A D L=$ instrumental activities of daily living; $S e$ in parentheses; Robust standard errors in parentheses, ${ }^{* * *} p<.01,{ }^{* *} p<.05,{ }^{*} p<.10$, CLHLS, 2008 and 2018.

Table 6 Decomposition of the Difference in ADL/IADL between Wave 2008 and Wave 2018 into Components Attributable to Changing Characteristics and Changing Coefficients

\begin{tabular}{ccccccc}
\hline \multicolumn{7}{c}{ Male } \\
\hline & \multicolumn{7}{c}{ ADL } & \multicolumn{2}{c}{ IADL } \\
\cline { 2 - 7 } & Coef. (\%) & $95 \%$ CI & Pct. & Coef. (\%) & $95 \%$ CI & Pct. \\
\hline Due to difference in E & -1.38 & $-3.05-0.28$ & 45.7 & $-2.45^{* * *}$ & $-3.96--0.93$ & 44.4 \\
Due to difference in C & -1.65 & $-8.36-5.07$ & 54.4 & -3.06 & $-13.27-7.16$ & 55.6 \\
Difference in Total & -3.03 & $-9.43-3.37$ & 100.0 & -5.50 & $-15.73-4.72$ & 100.0 \\
\hline \multirow{2}{*}{ Due to difference in characteristics (E) } & & \\
\hline \multirow{2}{*}{ Somatic comorbidities } & -0.04 & $-0.56-0.47$ & 1.5 & $0.39^{*}$ & $-0.02-0.80$ & -7.1 \\
Underweight & $-1.60^{*}$ & $-3.39-0.19$ & 52.8 & $-3.38^{* * *}$ & $-4.71--2.04$ & 61.4 \\
Overweight & 0.03 & $-0.85-0.90$ & -0.8 & 0.32 & $-0.41-1.06$ & -5.9 \\
Depression & -0.01 & $-0.15-0.12$ & 0.5 & $-0.23^{* * *}$ & $-0.38--0.09$ & 4.2
\end{tabular}




\begin{tabular}{|c|c|c|c|c|c|c|}
\hline Oldest-old & $0.25 * * *$ & $0.10-0.40$ & -8.3 & $0.45 * * *$ & $0.25-0.66$ & -8.2 \\
\hline \multicolumn{7}{|c|}{ Due to difference in coefficients (C) } \\
\hline Somatic comorbidities & -5.58 & $-25.50-14.34$ & 184.1 & -26.07 & $\begin{array}{c}-164.23- \\
112.09\end{array}$ & 473.8 \\
\hline Underweight & 1.91 & $-3.33-7.15$ & -62.9 & 7.07 & $-30.64-44.77$ & -128.4 \\
\hline Overweight & 4.86 & $-14.69-24.40$ & -160.3 & 12.24 & $-53.35-77.82$ & -222.3 \\
\hline Depression & -1.35 & $-6.18-3.48$ & 44.6 & 4.93 & $-21.93-31.79$ & -89.6 \\
\hline Oldest-old & 0.45 & $-1.19-2.08$ & -14.8 & 0.21 & $-4.94-5.37$ & -3.9 \\
\hline Intercept & -1.93 & $-15.69-11.84$ & 63.6 & -1.43 & $-45.67-42.81$ & 26.0 \\
\hline \multicolumn{7}{|c|}{ Female } \\
\hline & \multicolumn{2}{|c|}{$\mathrm{ADL}$} & \multicolumn{4}{|c|}{ IADL } \\
\hline & Coef. $(\%)$ & $95 \% \mathrm{CI}$ & Pct. & Coef. $(\%)$ & $95 \% \mathrm{CI}$ & Pct. \\
\hline Due to difference in $\mathrm{E}$ & $-1.95 * *$ & $-3.58--0.33$ & 71.4 & -1.34 & $-3.28-0.60$ & -16.9 \\
\hline Due to difference in $\mathrm{C}$ & -0.78 & $-6.49-4.93$ & 28.6 & $9.24 *$ & $-0.06-18.55$ & 116.9 \\
\hline Difference in Total & -2.73 & $-7.83-2.37$ & 0.0 & 7.90 & $-1.18-16.99$ & 0.0 \\
\hline \multicolumn{7}{|c|}{ Due to difference in characteristics (E) } \\
\hline Somatic comorbidities & $-0.40 * *$ & $-0.72--0.08$ & 14.8 & -0.44 & $-0.99-0.11$ & -5.6 \\
\hline Underweight & $-0.65 * *$ & $-1.16--0.14$ & 23.8 & $-1.15 * * *$ & $-1.93--0.37$ & -14.6 \\
\hline Overweight & -0.37 & $-1.43-0.69$ & 13.5 & -0.02 & $-1.42-1.37$ & -0.3 \\
\hline Depression & $-0.83^{*}$ & $-1.70-0.04$ & 30.3 & -0.53 & $-1.59-0.53$ & -6.7 \\
\hline Oldest-old & $0.30 * * *$ & $0.16-0.43$ & -11.0 & $0.81 * * *$ & $0.52-1.09$ & 10.2 \\
\hline \multicolumn{7}{|c|}{ Due to difference in coefficients (C) } \\
\hline Somatic comorbidities & 3.87 & $-42.63-50.38$ & -141.7 & -5.09 & $-15.12-4.94$ & -64.4 \\
\hline Underweight & -0.44 & $-5.77-4.88$ & 16.2 & 0.57 & $-2.79-3.92$ & 7.2 \\
\hline Overweight & 1.76 & $-18.79-22.31$ & -64.4 & -0.01 & $-8.26-8.24$ & -0.1 \\
\hline Depression & 0.23 & $-2.86-3.33$ & -8.5 & -2.69 & $-7.27-1.88$ & -34.1 \\
\hline Oldest-old & 0.20 & $-1.84-2.23$ & -7.2 & -0.22 & $-2.68-2.23$ & -2.8 \\
\hline Intercept & -6.40 & $-78.27-65.46$ & 234.2 & 16.70 & $0.01-33.38$ & 211.2 \\
\hline
\end{tabular}

Note. somatic comorbidities $=$ at least two additional somatic comorbidities from a list of 14 chronic somatic diseases; $\mathrm{E}=$ Characteristics; $\mathrm{C}=$ coefficients; Coef. $(\%)=$ coefficients multiplied with $100 ; \mathrm{CI}$ = confidence interval; Pct. = expressed as percentage; $A D L=$ activities of daily living; $I A D L=$ instrumental activities of daily living; **p <.05; ***p <.01; *p <.10, CLHLS, 2008 and 2018.

\section{Discussion}

Similar to the rising trend of chronic diseases in the world, chronic diseases in the elderly in China have exhibited a trend of rapid growth in general. In particular, the continuous rise in the incidence of diabetes and its complications poses a greater challenge to the improvement of the health of the elderly. many studies in China have shown that due to the implementation of health policy measures, the elderly disability rate has a trend of decline.

\section{Changes of diabetes prevalence in the elderly}

In 2020, the total number of diabetics in China has reached 130 million and The prevalence of diabetes among Chinese adults is $12.8 \%$ and family history of diabetes, advanced age, male, overweight, obesity and central obesity were the main risk factors for diabetes (Li Y,2020). This 
study also found that the prevalence of diabetes among the elderly above 65 in China increased from $4.2 \%$ in 2008 to $13.5 \%$ in 2018 , with a larger increase among women, and the prevalence of diabetes in the elderly was lower than that in the younger elderly. Using data obtained from the China Health and Retirement Longitudinal Study (CHARLS), another nationally representative survey, Zhong et al. [25. found the prevalence of self-reported diabetes among middle-aged and elderly Chinese adults was $8.4 \%$ in 2015 .

Diabetes prevalence may also be closely related to screening rates for diabetes. Diabetes screening rates were low in 2008, but have improved dramatically by 2018. Using blood diagnosis, $\mathrm{Xu}$ et al. found that the prevalence of diabetes in elderly people aged 60 and above in China reached $20.4 \%$ in 2008 [10. ], which was higher than the self-reported prevalence of diabetes in elderly people aged 65 as calibrated in this paper. Therefore, at least part of the reason was that the more extensive diabetes screening reduced the proportion of undiagnosed diabetes patients. The estimated standardized prevalence of diagnosed and undiagnosed diabetes in the Chinese adult population in 2013 was $10.9 \%$, with $4.0 \%$ diagnosed [11. ]. The rising prevalence of diabetes can be closely linked to dietary habits and unhealthy lifestyles. At the same time, the enhancement of diagnostic methods and access to medical services for diabetes has greatly improved the screening rate of diabetes.

\section{Diabetic patients have difficult changes in ADL and IADL}

In this study, it was found that the predictive probability of ADL difficulty in elderly diabetic patients decreased, the predictive probability of IADL difficulty in elderly diabetic patients increased slightly, and the predictive probability of IADL difficulty in elderly diabetic patients decreased somewhat. The results suggest that while the prevalence of diabetes has increased over time in the elderly, the rates of severe disability have decreased. In contrast, the predicted probability of difficulty in ADL and IADL increased slightly in people without diabetes. To a large extent, it reflects the positive effect of health management in patients with diabetes, while the disability of people without diabetes may be affected by other factors. To a certain extent, it conforms to the characteristics described by the "dynamic equilibrium hypothesis" for diabetics. The results suggest that the differences in difficulty with ADL between non-diabetics and diabetics are narrowing, as are men's IADL, but the differences in difficulty with IADL among women are widening, putting women with diabetes at a greater disadvantage.

Changes in comorbidities in diabetic patients and their contribution to increased ADL and IADL difficulties

A study of comorbidities in diabetic patients found that there was a slight but not statistically significant increase in the level of at least two somatic comorbidities in elderly Chinese men with diabetes during the 10-year period from 2008 to 2018. This conclusion means that in China, due to the small increase in the incidence of comorbidity of elderly diabetes, there is no significant influence on the decline of disability in elderly diabetes patients from 2008 to 2018, which may be related to the important influence of diabetes on elderly disability, reducing the influence of other comorbidity factors on disability. The relationship between comorbidities and disability in elderly diabetic patients in China shows more complex characteristics. This is consistent with the conclusion of complex relationship between comorbidities and disability reported in some studies $[2 ., 26$. .

The weight of diabetes patients has been increasing, with the proportion of underweight decreasing, but the proportion of overweight increasing. Although many studies have shown that a significant increase in obesity rate will increase the risk of disability [27. ]. Other studies have found 
a "U" or "J" shape in the relationship between weight or BMI and disability, meaning that not only are obese, but underweight older adults are at increased risk for disability [27. -29. ]. Some studies in China have shown that being underweight increases the risk of disability [23. ]. This study found an increased risk of disability in underweight diabetics, but did not find an increased risk of disability in normal-weight and some overweight elderly diabetics.

Many studies have shown that the co-existence of diabetes and depression has synergistic effects on the risk of disability and other adverse health outcomes [30. ]. We found that there was a decrease in the proportion of negative emotions in people with diabetes, which somewhat reduced the risk of disability.

Decomposition analysis showed that three comorbidities of diabetes, namely, somatic comorbidities, underweight and negative emotions, could decrease the proportion of ADL difficulty in elderly diabetic patients. For men and women, the biggest reason was the positive effect of a decrease in underweight and a decrease in negative emotions, respectively. The decrease of IADL in men was mainly due to the above two factors, while the increase of somatic comorbidities would lead to a slight increase in IADL incapacity. Unlike men, there was an upward trend in the proportion of women with difficulty with IADL due to other unexplained factors, despite the positive effect of the decrease in the proportion of underweight women. No significant overweight factor was found to contribute to the decrease in ADL or IADL in diabetic patients in this study.

Our results suggest that although the prevalence of underweight and negative mood in diabetic patients decreased over time, the negative effects of somatic comorbidities and advanced age on disability were somewhat suppressed. Although being overweight was not found to have a significant effect, it may increase the risk of disability as obesity rates rise among diabetics. At the very least, however, this study confirms that weight loss due to complications of diabetes may be associated with greater disability. This has important implications for the health management of patients with diabetes, not only to prevent the negative effects of obesity due to weight gain, but also to overcome the impact of underweight due to complications of diabetes on the risk of disability. At the same time, stimulating positive emotions can improve negative effects such as depression due to diabetes, which provides a clearer route for effective health management practices of diabetes in the elderly.

\section{Limitations}

This study has some limitations. Firstly, CLHLS individuals collect partial blood data, it does not include all respondents. We only use data from older adults who self-report whether they have diabetes. We do not use data from older adults who have been previously diagnosed with diabetes by a doctor, so we do not consider respondents that may have diabetes but do not or do not remember being diagnosed with diabetes. This can lead to a bias in diabetes prevalence.

Secondly, this study made no difference between type 1 and type 2 diabetes. Some scholars suggest using the onset age, more than 30 years as a threshold for type 2 diabetes cases to define with diabetes [6. ]. In order to avoid possible data bias, this study did not distinguish type 1 and type 2 diabetes, which may be a problem because comorbidities have different impact on disability of elderly with different types. In addition, the list of chronic diseases on which comorbidities of diabetics are based does not include all related diseases and therefore does not provide complete portfolios of their physical diseases.

Thirdly, we tried to analyze the survey data in 2014 to accurately analyze the disability trends of elderly with diabetes. However, since the follow-up survey was mainly conducted in 2014, it was 
not fully representative, and the analysis results showed large errors. Therefore, the time span of the final analysis is as long as 10 years, which may conceal more complex disability trends in Chinese elderly diabetes patients.

\section{Conclusion}

Our findings support the "dynamic equilibrium" hypothesis of incidence, suggesting that although the prevalence of diabetes increases with time, the proportion of ADL and IADL difficulties decreases with time, except for women with IADL difficulties. Although the degree of influence differed between men and women, it was confirmed that the decrease in underweight and negative emotions had a significant positive effect on the decrease in disability rates, but the increase in somatic comorbidities had a negative effect.

\section{Abbreviations}

CLHLS: Chinese Longitudinal Healthy Longevity Survey; ADL: Activities of daily living; IADL: Instrumental activities of daily living; BMI: Body mass index; PRC: People's Republic of China; CHARLS: China Health and Retirement Longitudinal Study; MDNRM: Multivariate decomposition for nonlinear response models

\section{Acknowledgements}

We appreciate some valuable suggestions from Guangzhou Wang, Institute of Population and Labor Economics, Chinese Academy of Social Sciences.

\section{Funding}

This study was supported by "Humanities and Social Science Research Project of Ministry of Education" (No. 20YJAZH091) and "Social Science Planning Project of Shandong Province" (No. 18CCZJ20).

\section{Availability of data and materials}

Please contact author for data requests or access Peking University Open Research Data Platform (https://opendata.pku.edu.cn).

\section{Authors' contributions}

SHL wrote the manuscript and performed the statistical analysis. CLS revised the manuscript and was responsible for the design of the study. Both authors read and approved the final manuscript.

\section{Ethics approval and consent to participate}

The survey was conducted with oral informed consent and the approval of the ethics committee of the University, in compliance with the principles of the Declaration of Helsinki. The authors take responsibility for results involving human subjects in this study.

\section{Consent for publication}

Not applicable.

\section{Competing interests}

The authors declare that they have no competing interests.

\section{Reference}


1. The Lancet. Latest global disease estimates reveal perfect storm of rising chronic diseases and public health failures fuelling COVID-19 pandemic. News Release 15-OCT-2020. https://www.eurekalert.org/pub releases/2020-10/tl-pss 101320.php, (accessed on 20 February 2021).

2. Jindai K, Nielson CM, Vorderstrasse BA. Multimorbidity QAR, Functional Limitations Among Adults 65 or Older. NHANES 2005-2012. Prev Chronic Dis. 2016;13: E151.

3. Lafortune, G. and G. Balestat (2007). Trends in Severe Disability Among Elderly People: Assessing the Evidence in 12 OECD Countries and the Future Implications. OECD Health Working Papers, No. 26.

4. Manton, K (2008), Recent declines in chronic disability in the elderly population: risk factors and future dynamics. Annual Review of Public Health. 29: 91-113.

5. Bleijenberg N, Zuithoff NPA, Smith AK, de Wit NJ, Schuurmans MJ. Disability in the individual ADL, IADL, and mobility among Older adults: a prospective cohort study. J Nutr Health Aging. 2017; 8:897-903.

6. Sperlich, Stefanie \& Beller, Johannes \& Epping, Jelena \& Safieddine, Batoul \& Tetzlaff, Juliane \& Geyer, Siegfried. Are Disability Rates among People with Diabetes Increasing in Germany? A Decomposition Analysis of Temporal Change between 2004 and 2015. Journal of Aging and Health. 2020; 0:1-12.

7. Gruenberg, E. M. The failures of success 1977. Milbank Quarterly. 2005; 4:779-800.

8. Hou, C. B., Ma, Y., Yang, X. H., Tao, L. X., Zheng, D. Q., Liu, X. T., . . Guo, X. H.. Disability Transitions and Health Expectancies among Elderly People Aged 65 Years and Over in China: A Nationwide Longitudinal Study. Aging and Disease. 2019; 6:1246-1257.

9. Yang W, Lu J, Weng J, et al. Prevalence of diabetes among men and women in China. N Engl J Med. 2010; 12:1090-1101.

10. Xu, Yu.; Wang, L.; He, J.; Bi, Y.; Li, M.; Wang, T.; Wang, L.; Jiang, Y.; Dai, M.; Lu, J.; et al. Prevalence and control of diabetes in Chinese adults. Jama. 2013; 310: 948-959.

11. Wang L, Gao P, Zhang M et al. Prevalence and ethnic pattern of diabetes and prediabetes in China in 2013. JAMA. 2017; 317:2515-2523.

12. Li Y, Teng D, Shi X, et al. Prevalence of diabetes recorded in China using 2018 diagnostic criteria from the American Diabetes Association: national cross sectional study. BMJ. 2020;369:m997.

13. The State Council of the People's Republic of China. Opinions on the Implementation of the Healthy China Initiative. http://www.gov.cn/zhengce/content/2019-07/15/content 5409492.htm. 2021-02-19 accessed.

14. Zhang,WJ, Wei, M. Disability level and time estimation of the elderly in China: an analysis based on pooled data. Demography Research. 2015; 05: 3-14.

15. Zeng Y, Feng Q, Hesketh T, Christensen K, Vaupel JW. Survival, disabilities in activities of daily living, and physical and cognitive functioning among the oldest-old in China: a cohort study. Lancet. 2017; 389:16191629.

16. Ding Hua, Yan Jie. Estimation and Trend of Disability Rate of the Elderly in China. China Population Science. 2018; 03:97-108.

17. Li, Z. H., Lv, Y. B., et. Al. Trends in the Incidence of Activities of Daily Living Disability Among Chinese Older Adults From 2002 to 2014. Journals of Gerontology Series a-Biological Sciences and Medical Sciences. 2020; 11:2113-2118.

18. Manton, K. G. Changing concepts of morbidity and mortality in the elderly population. The Milbank Memorial Fund Quarterly. Health and Society. 1982; 2:183-244.

19. Zeng, Y., Vaupel, J.W., Xiao, Z.Y., Zhang, C.Y., Liu, Y. Z,. The Healthy Longevity Survey and the Active Life Expectancy of the Oldest Old in China. Population: An English Selection. 2001; 1: 95-116.

20. Katz S., Ford A.B., Moskowitz R.W., Jackson B.A. and Jaffe M.W. Studies of illness in the aged. The index of ADL: A standardized measure of biological and psychosocial function. Journal of the American Medical Association. 1963; 185:914-919. 
21. Lawton MP, Brody EM. Assessment of older people: self-maintaining and instrumental activities of daily living. Gerontologist. 1969; 3:179-86.

22. Gao, M., Sa, Z., Li, Y. et al. Does social participation reduce the risk of functional disability among older adults in China? A survival analysis using the 2005-2011 waves of the CLHLS data. BMC Geriatr. 2018; 18:224.

23. Lv, Y.-B., Yuan, J.-Q., Mao, C., Gao, X., Yin, Z.-X., Kraus, V. B., . . Shi, X.-M.. Association of Body Mass Index with Disability in Activities of Daily Living Among Chinese Adults 80 Years of Age or Older. Jama Network Open, 2018; 1(5).

24. Powers DA, Yoshioka H, Yun M-S. Mvdcmp: Multivariate decomposition for nonlinear response models. Stata Journal. 2011; 11:556-576.

25. Zhonghua Wang, Xiangjun Li, Mingsheng Chen. Socioeconomic Factors and Inequality in the Prevalence and Treatment of Diabetes among Middle-Aged and Elderly Adults in China. Journal of Diabetes Research, 2018;1-12.

26. Calderón-Larrañaga, A., et al., Multimorbidity and functional impairment: bidirectional interplay, synergistic effects and common pathways. Journal of Internal Medicine. 2019; 3:255-271.

27. Chen H, Guo X. Obesity and functional disability in elderly Americans. J Am Geriatr Soc. 2008; 56:689-694.

28. Al Snih, S, Ottenbacher, KJ, Markides, KS, Kuo, YF, Eschbach, K, Goodwin, JS. The effect of obesity on disability vs mortality in older Americans. Arch Intern Med 2007; 167: 774-80.

29. Alley, DE, Chang, VW. The changing relationship of obesity and disability, 1988-2004. JAMA 2007; 298: 2020-2027.

30. Zhang Y, Ting RZ, Yang W et al. Depression in Chinese patients with type 2 diabetes: associations with hyperglycemia, hypoglycemia, and poor treatment adherence. J Diabetes. 2015; 7:800-808. 


\section{Figures}

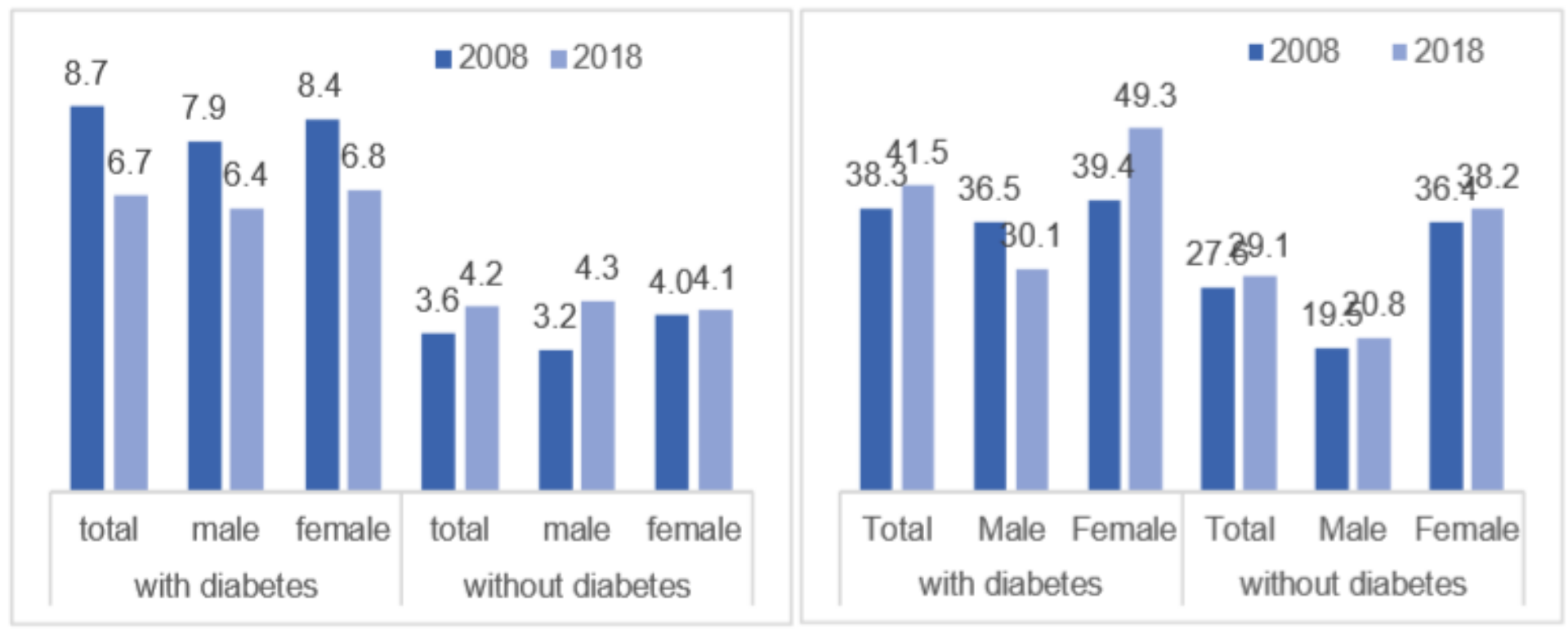

Figure 1

Change in ADL and IADL between 2008 and 2018 among people with and without diabetes, predicted probabilities in \%. Adjusted for age, education, married status and residence, for the bars "total" also for gender, CLHLS, 2008 and 2018. ADL = activities of daily living. IADL = instrumental activities of daily living 